\title{
Yale Child Study Center Autism Program
}

Published online: 3 June 2011

(c) Springer Science+Business Media, LLC 2011

10th Annual Summer Institute on Autism Spectrum Disorders

Monday-Thursday

July 11-14th, 2010

Yale School of Medicine, Harkness Auditorium, 333 Cedar Street, New Haven, CT

The Yale School of Medicine and Yale Child Study Center have scheduled this conference to be held on July 11-14, 2011 at The Yale School of Medicine Harkness Auditorium. The institute will highlight faculty from the Yale Child Study Center, as well as from the Center for Children with Special Needs. The Yale School of Medicine designates this educational activity for a maximum of 25 AMA PRA Category 1 Credits $^{\text {TM }}$.

Autism Spectrum Disorders (ASDs) are characterized by early onset socialization and communication impairments, as well as patterns of restricted interests and behavioral rigidities, that affect individuals across their lifespan. The number of individuals with ASD obtaining cognitive levels at or above the normative range is increasing. Yet, many of these individuals still fail to translate their potential into adaptive, real-life skills.

This stresses the need for education of professionals, pediatricians, psychiatrists, geneticists, general practitioners, nurses, educators, speech pathologists, psychologists, social workers, occupational therapists, physical therapists, and any professional in contact with children in a medical, clinical, or educational setting.

Topics will include:

- Clinical Assessment \& Evaluation of ASD

- Behavioral Assessment and Intervention

- Autism in Infancy \& Early Childhood

- Treatment \& Educational Programming

To register and view the conference program, please visit Yale CME: www.cme.yale.edu (click on 'CME Conference Schedule \& Registration') or call 203-785-4578.

For information regarding the Yale Autism Program, please visit http://www.autism.fm. 